\title{
Analysis of Intermodulation Distortion in OFDM Based Transmitter Using EER Technique
}

\author{
Stepan MATEJKA \\ Dept. of Radio Engineering, Czech Technical University in Prague, Technická 2, 16627 Prague 6, Czech Republic \\ matejka@fel.cvut.cz
}

Manuscript received June 22, 2015

\begin{abstract}
During the last two decades, new digital modulation systems have appeared in the audio broadcasting. Such broadcasting systems require new transmitters' concepts to enable the transmission of digitally modulated signals. Moreover, the selected modulation schemes (e.g. orthogonal frequency division multiplexing) require a high linearity power stage, which typically exhibits low efficiency due to high peak-to-average power ratio of the modulated signal. One of the promising transmitter concepts is the Kahn envelope elimination and restoration technique, where the original Cartesian in-phase and quadrature baseband signals are transformed to the envelope and phase signals. The main advantage of this technique is an ability to employ suitable types of highly efficient amplitude modulation transmitters for envelope amplification, while the phase modulated carrier is produced by an additional phase modulator. The substantial drawback of envelope elimination and restoration is nonideal recombination of linearly distorted amplitude signal and phase modulated carrier at the output power stage.
\end{abstract}

The aim of this paper is twofold. Firstly, to analyze the effect of the envelope and phase signals bandwidth limitation on the modulated signal in-channel distortion and out-of-channel emission. Secondly, to present the performance results as a reference for transmitter designers to properly set the envelope and phase paths to reach required in-channel signal quality and suppress out-of-channel products.

\section{Keywords}

Envelope Elimination and Restoration (EER), Orthogonal Frequency Division Multiplex (OFDM), transmitter, amplitude and phase modulation, intermodulation distortion, Error Vector Magnitude (EVM)

\section{Introduction}

New radio broadcasting standards operating in the LW, MW, and SW broadcasting bands, such as Digital Radio Mondiale (DRM) [1] and HD Radio [2], are mainly based on the orthogonal frequency division multiplexing (OFDM) modu- lation scheme. The implementation of such new OFDM based schemes caused the new high-power transmitter designs to evolve during the past two decades. It is not only the demand for implementation of the new digital modulation schemes, but also the low efficiency of traditional linear amplifiers due to a high peak-to-average power ratio (PAPR) [3], which moves the traditional linear power stage transmitter construction to the high-power switching conception [4-6]. Nowadays, the pulse duration modulation (PDM) and its special case - pulse width modulation (PWM) - are the most common modulation techniques used in the new high-power AM transmitters [7], [8]. Utilizing the envelope elimination and restoration (EER) modulation method proposed by Kahn in 1952 [9], it is possible to design a switching transmitter convenient for digital broadcasting (e.g. DRM 30), which is still based on the PWM technique applied to the transmitter envelope signal processing path.

The EER technique is also known as the polar modulation technique. In its basic arrangement, the amplitude and phase informations are separated from the original lowlevel modulated signal using an envelope detector and a hard limiter. The rectangular-shaped phase modulated carrier is then amplified by a highly efficient switching power amplifier such as class D, E, or F. The amplitude (envelope) signal controls the power supply voltage of the phase signal power amplifier. In this way, the power amplifier works as a time domain multiplier. Thus the separated amplitude and phase signals recombine and the power amplifier produces amplified modulated signal. As a switching mode power amplifier can be used in the power supply block as well, the overall transmitter efficiency achieves very good values.

The EER transmitter architecture is attractive not only for high PAPR narrowband communication systems but also for wideband systems [10], [11]. Increasing envelope amplifier bandwidth, however, means increasing the negative influence of switching characteristics of power transistors on the total distortion of the power amplifier. Although a large effort has been devoted to the investigation of EER enhancement [12], [13], it still holds that this undesirable effect of the wideband EER leads either to the construction of EER based amplifiers with only a low output power (e.g. WLAN 802.11 standard families), or to use other techniques to reach high 


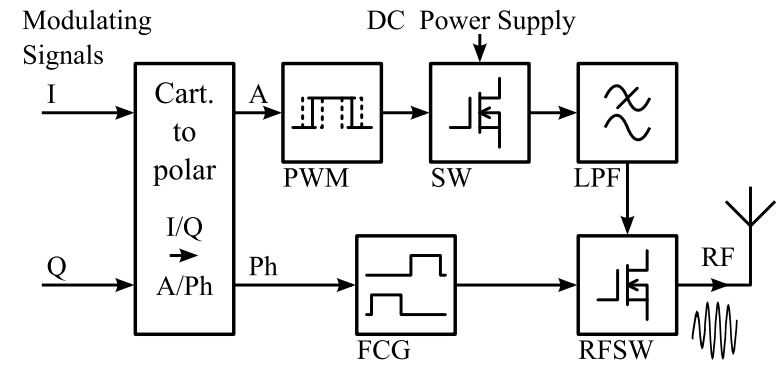

Fig. 1. Generalized block diagram of a PWM based switching transmitter for OFDM modulation scheme.

linearity and high power efficiency (e.g. Doherty amplifier for COFDM broadcasting standards [14]).

The simplified block diagram of a typical PWM based switching transmitter for OFDM modulation scheme following the Kahn EER modulation method is shown in Fig. 1. The Cartesian modulating signals - digital in-phase (I) and quadrature (Q) signals from IFFT based OFDM modulator - are converted to their amplitude (A) and phase $(\mathrm{Ph})$ equivalents by means of the specialized digital signal processing algorithm (e.g. CORDIC, [15]) with simultaneous sampling rate conversion (up-sampling) due to increased amplitude and phase signals bandwidth. The amplitude baseband signal feeds the envelope path of the transmitter, while the phase baseband signal feeds the carrier frequency path. The main building blocks of the envelope path are a low-level PWM generator (PWM) driving a class-D switching amplifier (SW), which produces a high-level PWM pulse train proportional to the modulating signal amplitude, and a lowpass filter (LPF), which rejects unwanted frequency components to reconstruct amplified amplitude signal. The carrier path consists of a carrier frequency generator (FCG), which generates phase modulated carrier signal, followed by an inverter (RFSW) powered from the envelope path LPF. The inverter is typically constructed as a set of full-bridge MOSFET switching stages, where the outputs from all stages are summed, filtered, and matched to a connected transmitting antenna. The RFSW works as a combiner (multiplier), whose output produces desired high-power band-limited modulated signal.

Inside the real transmitter, the recombination of the amplitude signal and the phase modulated carrier to narrowband modulated signal is not perfect, which results in intermodulation distortion [16-18]. There are several effects producing both the in-channel distortion, which results in modulated signal Error Vector Magnitude (EVM) increase, and the outof-channel emission, which decreases not only the transmitter Adjacent Channel Power Ratio (ACPR), but produces wideband transmitter noise:

- Delay mismatch between the amplitude signal and the phase modulated carrier signal at the output power stage.

- Bandwidth limitation and non-constant group delay of the transmitter envelope path.
- Bandwidth limitation and undersampling effects of the carrier frequency phase modulator.

The first phenomenon, the delay mismatch, is well discussed in papers [19], [20]. In the contemporary transmitter designs, where the low-level signal processing is performed in the digital domain using the digital signal processors (DSP) or FPGA [21], [22], it is not problematic to compensate for such type of distortion.

The second phenomenon referred to as linear distortion is a result of filtering process in the digital part of the transmitter envelope section, the PWM modulator, and the low-pass LC filter, which works as a PWM demodulator generating a high-level amplitude signal. Due to its analog form, the low-pass LC filter exhibits not only the bandwidth limitation (it must reject PWM spectral components located at the vicinity of the PWM switching frequency multiples), but a non-linear phase of the transfer function resulting in a nonconstant group delay. Both effects can be corrected up to the LPF cut-off frequency, which is determined by the PWM switching frequency and by the PWM out-of-baseband spectral components rejection requirements. This effect is discussed in [19], [20] only partially and author focuses on the out-of-band distortion only.

The third phenomenon appears in the case of the carrier phase modulator working with a low sampling frequency. The best way is to implement a numeric modulator, which exactly calculates edge positions of the rectangular shaped carrier signal. This modulator in conjunction with direct carrier signal synthesis ensures low distortion of the phase modulated carrier.

In this paper, the effect of the amplitude and phase signal bandwidth limitation is studied and its impact on the modulated signal in-channel and out-of-channel distortion is described. The rest of the paper is organized as follows. In Sec. 2, a simplified mathematical model of the envelope and phase signal processing paths is proposed, some properties of the amplitude and phase signals are specified and the power spectral properties of both polar components are determined. The simulation results of the amplitude and phase signal bandwidth limitations are given in Sec. 3. Both effects - the out-of-channel emission and the in-channel distortion due to bandwidth limitations - are demonstrated. The simulation results are discussed and summarized in the form of transmitter design recommendations in Sec. 4, and the paper concludes in Sec. 5.

\section{Problem Statement}

The proposed simplified model of a transmitter based on the ERR technique is shown in Fig. 2, [21], where the band-limiting low pass filters for the amplitude and phase paths are added.

The input Cartesian in-phase and quadrature components, $i(t), q(t)$, produced by preceding baseband OFDM 


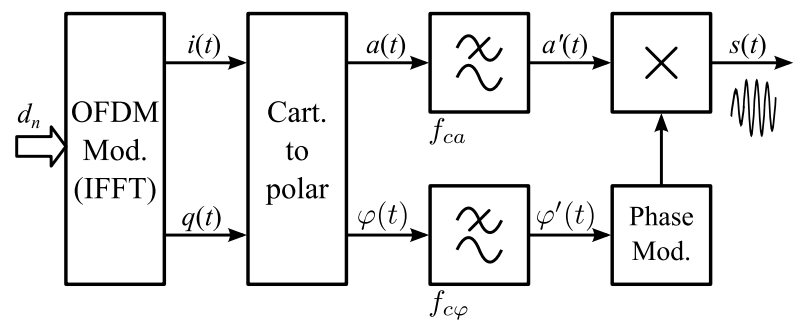

Fig. 2. Simplified model of EER transmitter.

modulator are fed into Cartesian to polar converter, which produces the amplitude and phase components, $a(t), \varphi(t)$. It is supposed that both the amplitude and phase components are produced without a distortion, following the formulas

$$
a(t)=\sqrt{i^{2}(t)+q^{2}(t)}
$$

and

$$
\varphi(t)=\operatorname{atan} 2(q(t), i(t))
$$

where $\operatorname{atan} 2(x, y)$ is the four-quadrant inverse tangent $\left(\tan ^{-1}\right)$ which returns values in the closed interval $[-\pi, \pi]$ based on the values of Cartesian coordinates $x$ and $y$.

At the final stage, the phase signal is converted to the phase modulated carrier and then multiplied by the amplitude signal. Thus the ideal modulated signal at the carrier frequency, $\omega_{c}$, is given by

$$
s(t)=a(t) \cos \left(\omega_{\mathrm{c}} t+\varphi(t)\right)
$$

and it is equivalent to the output of the ideal Cartesian form of a quadrature modulator defined as

$$
s_{\mathrm{c}}(t)=i(t) \cos \left(\omega_{\mathrm{c}} t\right)+q(t) \sin \left(\omega_{\mathrm{c}} t\right) .
$$

In a practical implementation, the amplitude signal is converted to a high-power PWM signal and filtered by a reconstruction low-pass LC filter. This PWM and LPF sections are preceded by a digital low-pass compensation filter (typically with high rate roll-off), which corrects the passband flatness and linearizes the phase. Hence, the amplitude path of the transmitter is modeled by an ideal LPF characterized by its cut-off frequency, $f_{\text {ca }}$, and the flat pass-band with a linear phase. Similar applies for the phase signal, where the filtering effect is inherent to signal calculation and/or resampling process. Note the cut-off frequency, $f_{c \varphi}$, of the phase path is generally different from $f_{\text {ca }}$. The output modulated signal changes to

$$
s(t)=a^{\prime}(t) \cos \left(\omega_{\mathrm{c}} t+\varphi^{\prime}(t)\right)
$$

where $a^{\prime}(t)$ and $\varphi^{\prime}(t)$ are the low-pass filtered amplitude and phase signals, respectively. It is known (see appendix of [9] or [23]), that both the amplitude and phase signals have widened power spectral densities (PSDs) in comparison with the frequency limited baseband Cartesian signals $i(t)$ and $q(t)$. Thus the signal processing, which inherently filters the amplitude and/or phase signals, linearly distorts these signals. Unfortunately, such type of distortion has considerable impact on a resultant modulated signal. To estimate the demands on the cut-off frequencies in both amplitude and phase paths, we can determine the key power spectral density (PSD) parameters of the original $a(t)$ and $\varphi(t)$ signals.

Assume that $i(t)$ and $q(t)$ are a band-limited independent real valued Gaussian processes with zero mean and the same autocorrelation function $R_{i}(\tau)=k \cdot \operatorname{sinc}\left(2 B_{\mathrm{C}} \tau\right)$, where $2 B_{\mathrm{C}}$ is the bandwidth of processes flat PSDs. We can split (1) to two consecutive operations - the power to 2 and the square root. The correlation function of $i^{2}(t)$ (and $\left.q^{2}(t)\right)$ is [24]

$$
R_{i i}(\tau)=R_{i}^{2}(0)+2 R_{i}^{2}(\tau)
$$

The PSD of $i^{2}(t), S_{i i}(f)$, is in accordance with WienerKhinchin theorem [25], [26] the Fourier transform of (6), i.e.

$$
S_{i i}(f)=c_{1} \cdot \delta(f)+c_{2} \cdot S_{i}(f) \otimes S_{i}(f)
$$

where $S_{i}(f)$ is PSD of $i(t)$. The $S_{i i}(f)$ contains two terms. The first term represents a DC component of squared $i(t)$; the second term represents the continuous PSD calculated as a single-folded convolution of $S_{i}(f)$. Thus the double-sided PSD of $i^{2}(t)$ is spread over $4 B_{\mathrm{C}}$ bandwidth with the component's maximal frequency $2 B_{\mathrm{C}}$. The sum of the $i^{2}(t)$ and $q^{2}(t)$ does not change the shape of PSD due to the same statistics but independence. The following square root operation is again non-linear and it may be represented by an infinite series over limited interval. In this case, it is possible to use a generalized form for the autocorrelation function of a stationary process passed through a non-linear time invariant system [27], and we obtain

$$
R_{a}(\tau)=k_{0}+\sum_{n=1}^{\infty} k_{n} R_{i}^{2 n}(\tau) .
$$

Applying Wiener-Khinchin theorem, we obtain

$$
S_{\mathrm{a}}(f)=w_{0} \cdot \delta(f)+\sum_{n=1}^{\infty} w_{n} \cdot S_{i}(f) \stackrel{2 n}{\otimes} S_{i}(f) .
$$

This expression has a simple physical interpretation. The power spectrum of the amplitude signal is composed of DC component and the sum of terms obtained by a $(2 n)$ th convolution of the $i(t)$ (or $q(t)$ ) PSD with itself. Due to the infinite number of terms, the power spectrum of the amplitude signal is unbounded, but with decreasing character in increasing frequency. Similar considerations can be done for the phase modulating signal, where, finally, PSD is again decreasing but unbounded along the frequency axis.

For illustration purposes, Fig. 3 shows the typical amplitude and phase baseband signals in the time-domain for OFDM with 205 carriers, 16-QAM modulation scheme (for the test signal details see next section). The amplitude signal is characteristic by sharp edges at the points where the signal value is close to zero. This is an effect of power and square root mathematical operations during Cartesian to polar conversion. Similarly, the phase signal exhibits distinct phase 

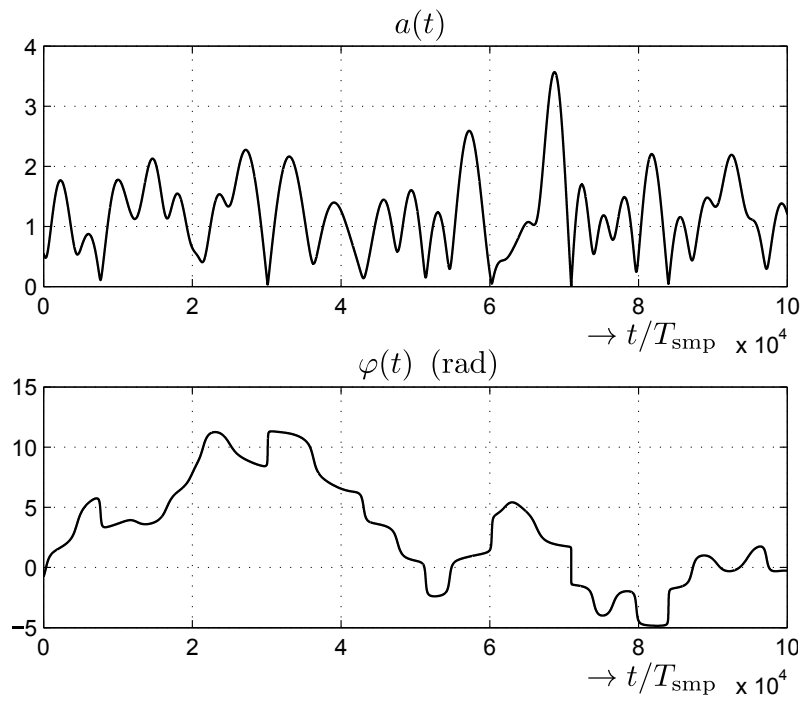

Fig. 3. Example of normalized amplitude, $a(t)$, and phase, $\varphi(t)$, signals of OFDM signal in the time domain (205 carriers, 16-QAM; similar to DRM 30 Mode A/16QAM). Time is normalized to a system sampling frequency, $T_{\text {smp }}$.

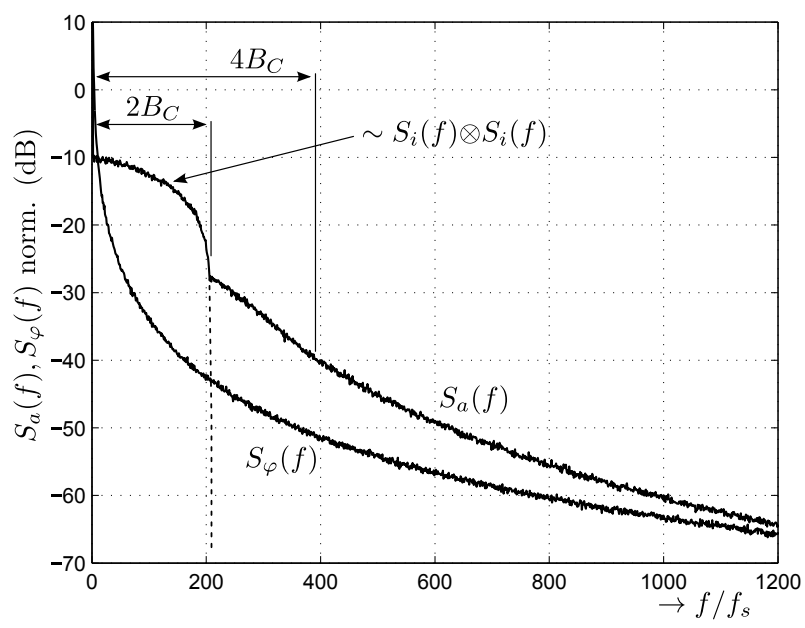

Fig. 4. Example of power spectra of amplitude, $S_{a}(f)$, and phase, $S_{\varphi}(f)$, signal of OFDM baseband signal ( 205 carriers, 16-QAM; similar to DRM Mode A/16-QAM). Frequency is normalized to OFDM carriers spacing, $f_{s}$.

transitions at these points with changes up to $\pm \pi$. These effects result in widening of the power spectral densities of both amplitude and phase signals as depicted in Fig. 4.

The filtering of the amplitude and/or phase signals within ERR technique always results in linear distortion. After the signals recombination at the power stage, which is highly non-linear operation, the in-channel distortion and the out-of-channel emission appear. The in-channel distortion decreases the signal-to-noise ratio and affects qualitative modulation parameters - the Error Vector Magnitude (EVM) or Modulation Error Ratio (MER). The out-of-channel emission also known as spectrum regrowth decreases the transmitter adjacent channel power ratio (ACPR) and disturbs the receivers working at the adjacent channels.

\section{Simulation}

The performance degradation of the modulated signal due to bandwidth limitations of the amplitude and phase modulating signals is evaluated in this section. The simulation results were obtained from Matlab following the model in Fig. 2 converted to a discrete form. The system sampling frequency is large enough $(\approx 5000$ times greater than the Cartesian components bandwidth) to ensure negligible noise floor resulted from aliasing of non-filtered components.

The DRM 30 [1] has been chosen as a reference OFDM system, since it is one of the most promising worldwide broadcasting systems intended for carrier frequencies up to $30 \mathrm{MHz}$, it is an open standard system, and the European Broadcasting Union counts DRM as Digital Radio system in Europe is substituting analogue broadcasting and complementing DAB for local broadcasts [28]. Since the generation of a complete DRM signal for radio interface performance tests is not necessary, only a simplified model of the test signal was used.

The test signal follows DRM system Mode A specification, where spectrum occupancy 2 has been used but with occupied unused carriers. All carriers are modulated by the same modulating scheme (16-QAM) with pseudorandom independent data symbols and the same average energy per symbol. The DRM 30 uses a guard interval consisting in a cyclic continuation of the useful signal part (the cyclic prefix) to overcome the multipath propagation at the receiver. This feature is not used in presented simulation. Each OFDM symbol in the time domain is produced as the inverse Fourier transform (IFFT) of a rectangular-shaped PSD without a noticeable power in the adjacent channels. The distorting low-pass filters for amplitude and phase paths are specified by cut-off frequencies $f_{c a}$ and $f_{c \varphi}$, respectively. The frequencies are mostly expressed as a multiple of the Cartesian components $i(t)$ and $q(t)$ bandwidth $B_{C}$. The LPF amplitude response equals 1 for $f<f_{c a}$ (or $f<f_{c \varphi}$ ) and zero elsewhere, the group delay is set to zero. Thus it forms ideal LPF (unless otherwise noted). The PSD of baseband equivalent modulated signal $S_{S}(f)$ was estimated by the periodogram power spectral estimator as an average over the interpolated set of OFDM symbols and is normalized to $0 \mathrm{~dB}$ within the channel bandwidth. The frequency axis is scaled in the OFDM subcarriers distance, $f_{s}$. The presented results are applicable to any OFDM system as long as the number of carriers is large enough to satisfy the $i(t), q(t)$ statistical characteristics.

Figure 5 outlines the spectrum regrowth of the modulated signal for different amplitude signal bandwidth limits; the phase modulating signal passes through without changes. Figure 6 shows the similar results when only the phase signal is limited instead the amplitude signal. Finally, the effect of the both amplitude and phase bandwidth limitation with the same cut-off frequencies is depicted in Fig. 7.

To analyze the effect of the LPF amplitude response in the transition area, the spectrum regrowth has been evaluated 


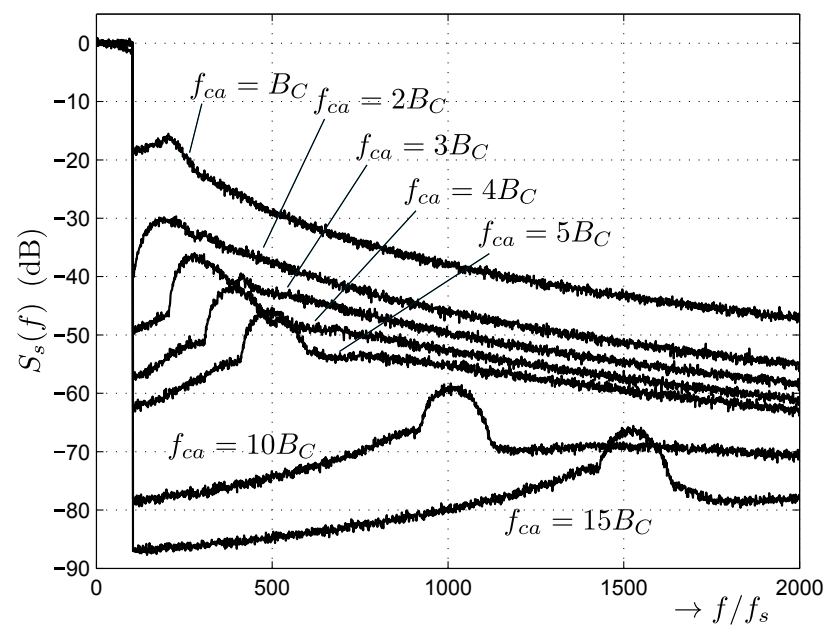

Fig. 5. Modulated output signal spectrum regrowth due to the bandwidth limitation of the amplitude signal.

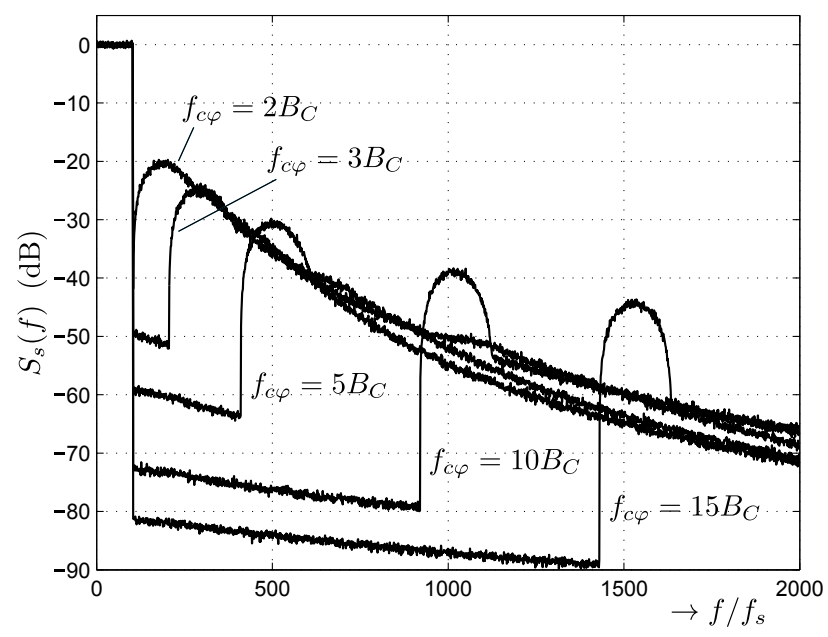

Fig. 6. Modulated output signal spectrum regrowth due to the bandwidth limitation of the phase signal.

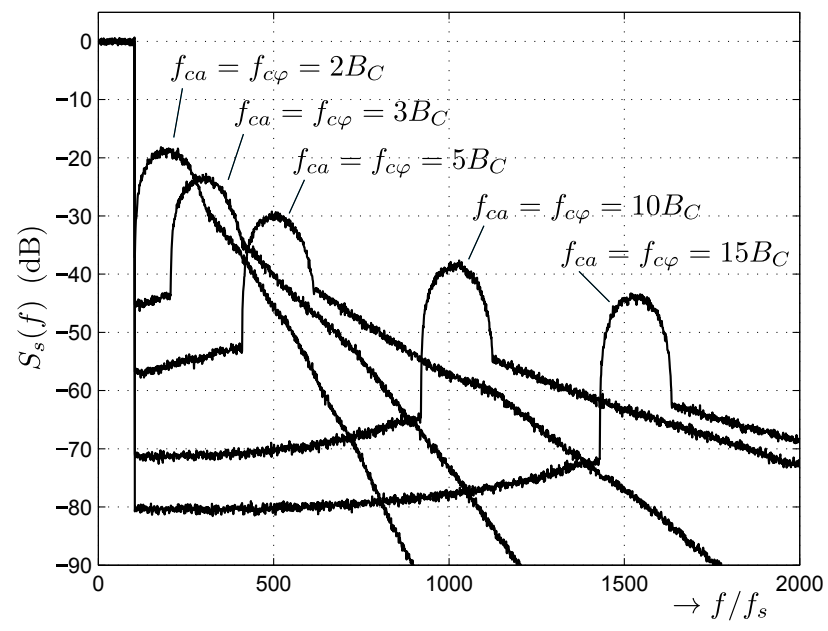

Fig. 7. Modulated output signal spectrum regrowth due to simultaneous bandwidth limitation of the amplitude and phase signals with the same cut-off frequencies.

for various rates of LPF roll-off at frequencies above the cut-off frequency. The results are outlined in Fig. 8 for two cut-off frequencies, $f_{\text {ca }}=2 B_{\mathrm{C}}, f_{\text {ca }}=5 B_{\mathrm{C}}$, and several different roll-off factors.

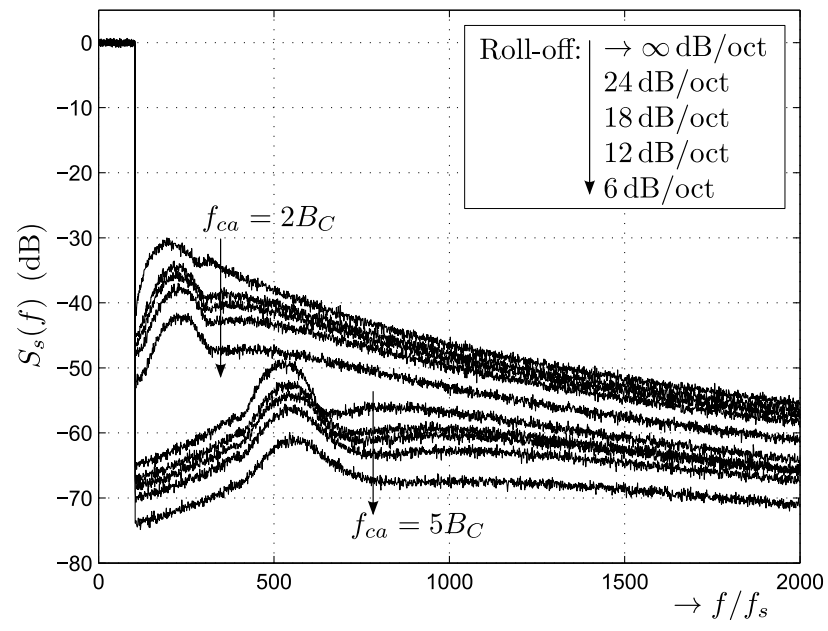

Fig. 8. Spectrum regrowth for different LPF roll-off factors. Only the amplitude signal is filtered.

The standard method to quantify a close out-of-channel emission in the digital communication systems is to specify the Adjacent Channel Power Ratio (ACPR), also known as Adjacent Channel Leakage Ratio (ACLR) and Alternate Channel Power Ratio (AltCPR). Both are the important metrics for communication systems to characterize spectrum regrowth simply by the ratio of the modulated signal power in an occupied channel versus the power emitted into an adjacent or alternate channel. Unfortunately, the ACPR and AltCPR are not specified in the ETSI DRM standards [29] and [30]. These standards only define measurements of the spurious emissions limited by mean power absolute levels or relative levels below the mean power supplied to the antenna port and out-of-band emissions limited by a spectral mask. In this paper, the ACPR definition follows Upper-Sideband

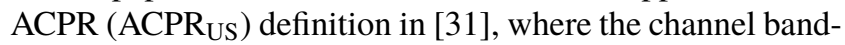
width is equal to $2 B_{\mathrm{C}}$ and there is no guard interval between adjacent channels, i.e.

$$
\mathrm{ACPR}=10 \log _{10}\left(\frac{\int_{f_{\mathrm{c}}-B_{\mathrm{C}}}^{f_{\mathrm{C}}+B_{\mathrm{C}}} S_{\mathrm{s}}(f) \mathrm{d} f}{\int_{f_{\mathrm{c}}+B_{\mathrm{C}}}^{f_{\mathrm{c}}+3 B_{\mathrm{C}}} S_{\mathrm{s}}(f) \mathrm{d} f}\right)
$$

where $S_{\mathrm{S}}(f)$ is the power spectral density of the modulated signal and $f_{\mathrm{c}}$ is the center frequency of the occupied channel. For AltCPR, the formula is the same, except we center the alternate channel $4 B_{\mathrm{C}}$ away from the OFDM center carrier frequency, i.e.

$$
\operatorname{AltCPR}=10 \log _{10}\left(\frac{\int_{f_{\mathrm{c}}-B_{\mathrm{C}}}^{f_{\mathrm{c}}+B_{\mathrm{C}}} S_{\mathrm{s}}(f) \mathrm{d} f}{\int_{f_{\mathrm{c}}+3 B_{\mathrm{C}}}^{f_{\mathrm{c}}+5 B_{\mathrm{C}}} S_{\mathrm{s}}(f) \mathrm{d} f}\right)
$$

Figures 9 and 10 show ACPR and AltCPR, respectively, for different amplitude and phase path LPF cut-off frequencies. 


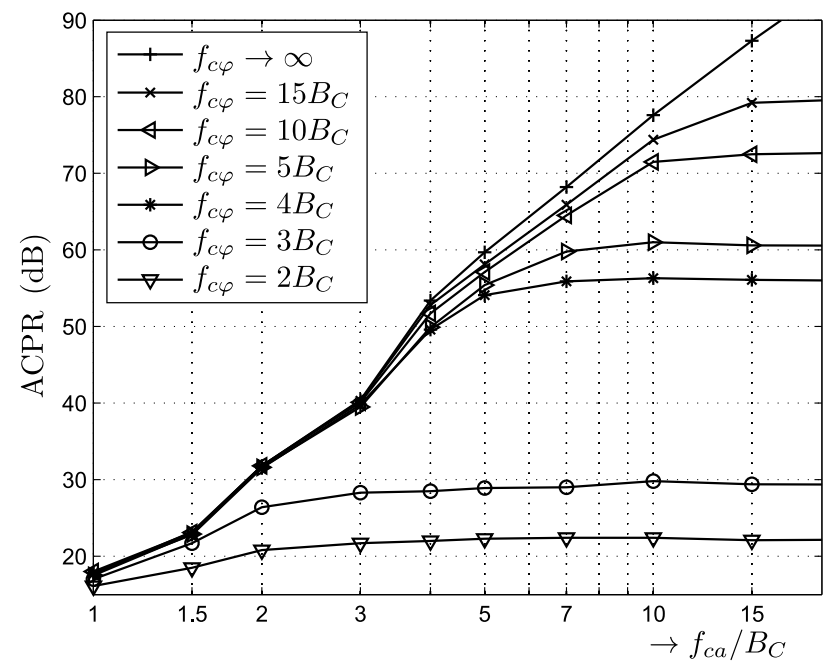

Fig. 9. Adjacent channel power ratio (ACPR) due to bandwidth limitation of amplitude signal and phase signal.

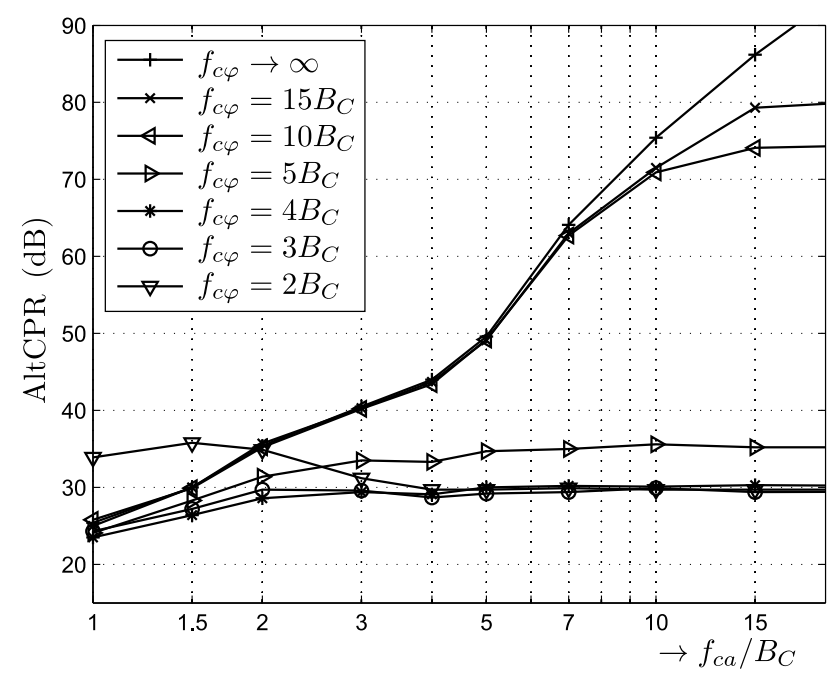

Fig. 10. Alternate channel power ratio (AltCPR) due to bandwidth limitation of amplitude signal and phase signal.

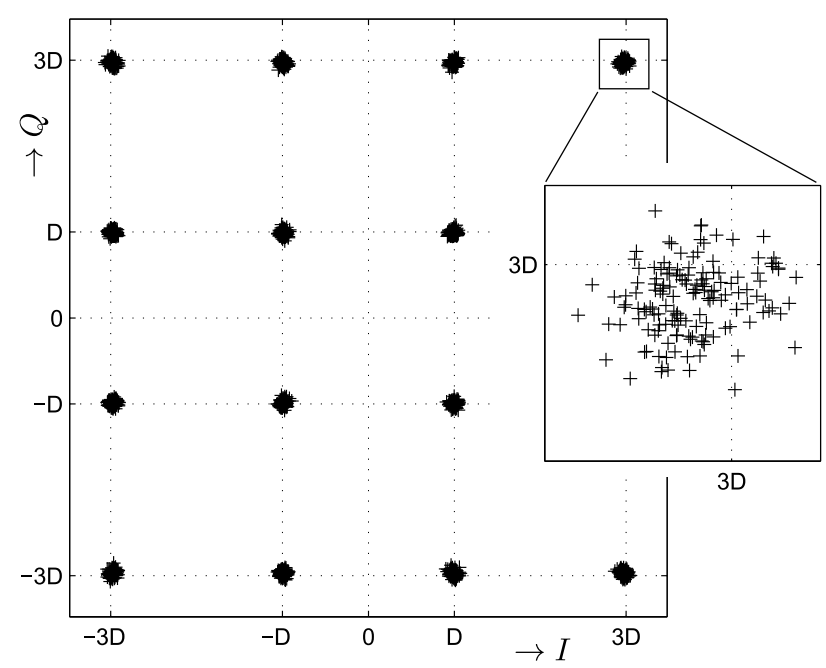

Fig. 11. In-channel modulated signal distortion - 16-QAM scatter plots for the complete carriers budget due to simultaneous bandwidth limitation of the amplitude and phase signal ( $D$ is the scale factor to set-up given energy per bit $E_{\mathrm{b}}$ at the transmitter, $\left.D=\sqrt{E_{\mathrm{b}} / 10}\right)$.

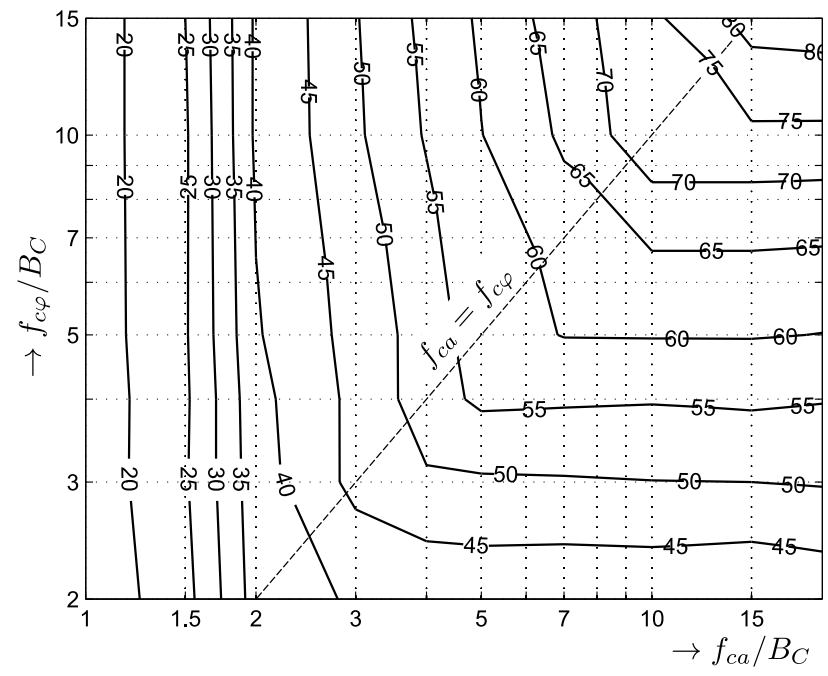

Fig. 12. In-channel modulated signal distortion (IEVM) due to simultaneous bandwidth limitation of the amplitude and phase signals.

The in-channel distortion due to imperfect amplitude and phase components recombined at the transmitter final stage results in Signal-to-Noise Ratio (SNR) degradation and, consequently, in the increase of the error vector in the modulation domain I-Q plane. The Error Vector Magnitude (EVM) is a measure which numerically describes the deviation of actual constellation points from the ideal locations in the constellation diagram. The root-mean-square value of EVM is evaluated according to

$$
\mathrm{EVM}_{\mathrm{RMS}}=\sqrt{\frac{\frac{\frac{1}{N} \sum_{n=1}^{N}\left|C_{0, n}-C_{\mathrm{M}, n}\right|^{2}}{\frac{1}{N} \sum_{n=1}^{N}\left|C_{0, n}\right|^{2}}}{}}
$$

where $C_{\mathrm{M}, n}$ is the $n$-th normalized measured constellation point within OFDM block, $C_{0, n}$ is the ideal normalized constellation point nearest to $C_{\mathrm{M}, n}$, and summation is taken over all occupied subcarriers within OFDM block. Due to the straightforward relation to SNR, the Inverse EVM (IEVM) was evaluated in this paper, expressed by

$$
\mathrm{IEVM}_{\mathrm{RMS}}=20 \log _{10}\left(\frac{1}{\mathrm{EVM}_{\mathrm{RMS}}}\right) .
$$

Note the distorted signal at the transmitter output suffers from signal compression (see Fig. 11). Therefore, the OFDM block scaling and rotation were performed before IEVM calculation. The simulated IEVM is outlined in Fig. 12 for different cut-off frequencies in both amplitude and phase paths.

\section{Results and Comments}

Based on the simulation results obtained in the previous section, several conclusions and recommendations can be made:

- The out-of-band emission power is monotonically but inversely proportional to the cut-off frequencies of the 
amplitude or phase path LPFs - the higher cut-off frequency, the lower emission power - see Fig. 5 and Fig. 6. Comparing both figures, it can be concluded that the modulated signal out-of-band emissions are very much alike (except the small cut-off frequencies $\leq 2 B_{C}$, where the modulated signal is more sensitive to the phase bandwidth limitation) in the band up to the cut-off frequency, however, the spectrum regrowth is more sensitive to the phase path cut-off frequency at the frequencies greater or equal to the cut-off frequency. The cause of this effect is twofold. Firstly, the frequent transitions of the phase signal generate ringing after passing through the LPF, which results in significant local extremes in modulated signal PSD located near the cut-off frequency exceeding the similar effect in the amplitude path. Secondly, the PSD of the phase signal is generally more flat than the PSD of the amplitude signal at the higher frequencies. Thus the non-linear process of recombination is more sensitive to the phase signal distortion. This effect is also noticeable in the scenario, where the cut-off frequencies - for the both amplitude and phase paths - are the same - see Fig. 7. It is then possible to observe (for the cut-off frequencies $\geq 3 B_{C}$ ) that at the frequencies lower than the cut-off frequency the amplitude and phase signals recombine and generate the spectrum regrowth at similar level as without either the phase path limit or the amplitude path limit (Figs. 5, 6). However, the spectrum regrows mainly due to the phase path limit at the frequencies close to the cut-off frequency. Finally, significant reduction of the out-of-channel emissions can be observed at the frequencies greater than the cut-off frequency as a result of simultaneous amplitude and phase signals bandwidth limitation.

- The effect of replacing the ideal LPF with their equivalent roll-off finite LPF is shown in Fig. 8. Only the amplitude path filtering is evaluated, the results and conclusions for the phase path are the same. It is clear that the use of the first order LPF with the roll-off $-6 \mathrm{~dB} /$ octave decreases the PSD outside the channel up to $10 \mathrm{~dB}$. Increasing the roll-off of the LPF approaches the PSD back to its original value obtained for the ideal LPF. Thus, there is not a significant benefit in reducing the slope of the LPF.

- Adjacent channel power ratio (ACPR) or alternate channel power ratio (AltCPR) (Fig. 9, Fig. 10) were evaluated for different amplitude path and phase path cut-off frequencies. There is considerable value change between the cut-off frequencies $3 B_{\mathrm{C}}$ and $4 B_{\mathrm{C}}$ (ACPR) and $5 B_{\mathrm{C}}$ and $10 B_{\mathrm{C}}$ (AltCPR), which is a consequence of the PSD increase at the vicinity of the cut-off frequency. Both the ACPR and AltCPR tend to limit for increasing cut-off frequency, $f_{\text {ca }}$. This is a result of the out-ofchannel emission predominantly caused by the phase bandwidth limit.
- To reach for example $60 \mathrm{~dB}$ out-of-channel emission attenuation (DRM 30 standard spectral mask, [1]), it is necessary to set the cut-off frequency to $10 B_{\mathrm{C}}$, i.e. approximately at $50 \mathrm{kHz}$ for DRM 30 Mode A $9 \mathrm{kHz}$ or $10 \mathrm{kHz}$ channel.

- The in-channel distortion gives optimistic results even for low cut-off frequencies within both amplitude and phase paths - see Fig. 12. The cut-off frequency equal $2 B_{\mathrm{C}}$ ensures IEVM better than $35 \mathrm{~dB}$ - i.e. the sufficient value for demodulation of the 64-QAM modulation scheme. The increase of the cut-off frequency results in approximately $15 \mathrm{~dB}$ per octave improvement of IEVM. Fig. 12 shows that the optimal cut-off frequencies settings in terms of their minimal product, $f_{\mathrm{ca}} \cdot f_{\mathrm{c} \varphi}$, is close to the line of equality $f_{\mathrm{ca}}=f_{\mathrm{c} \varphi}$. Further increase of $f_{\mathrm{ca}}$ or $f_{\mathrm{c} \varphi}$ does not significantly improve IEVM.

- The presented results are applicable not only for narrowband EER systems, but also for wideband EER systems under the assumption of Gaussian character of amplified signal with a frequency limited flat PSD. It should however be noted that the switching characteristics of transistors inside the switching power amplifier give rise to unwanted intermodulation products of comparable or even greater levels than products arising due to the bandwidth constraints of the system. This effect is serious in the case of wideband EER and may not be neglected.

- Note that presented results were obtained from the simulation of the OFDM signal without the cyclic prefix (and without the pilot subcarriers which use different modulation scheme than the data carriers). In this case it was possible to determine a very small value of the intermodulation products in the adjacent channels. For the OFDM with cyclic prefix, the intermodulation products close to the channel may be masked by sidelobes of the OFDM signal PSD. Furthermore, the OFDM signal PSD will exhibit the in-channel and the out-of-channel ripples. The ACPR, AltCPR, EVM, and IEVM as the integral quantities are not so sensitive to this ripple but are biased. Comparative test simulations with and without guard interval for a high level of intermodulation products showed, that it is necessary to consider presented values as the best case.

\section{Conclusions}

Given a simplified transmitter model based on the Kahn polar EER architecture, the effect of the internal amplitude and phase signals bandwidth limitation on the OFDM modulated signal at the transmitter output has been investigated. The analytical background was briefly reviewed to obtain basic characteristics of the PSD of the amplitude and phase signals. Throughout the problem analysis and simulation, 
the other signal processing impairments, e.g. the power amplifier non-linearity, the switching distortion, the PWM sticky effect, the LC filter group delay unevenness etc., were omitted to emphasize the effect of the signal bandwidth limitation only and to make the derivations simpler. Both the in-channel distortion, quantified as IEVM degradation, and the out-of-channel emission (PSD, ACPR, AltCPR) were tested for different bandwidth limiting LPF cut-off frequencies. The presented performance results can be used by transmitter developers to design the EER transmitter system to reach required in-channel intermodulation distortion and out-of-channel emission.

\section{Acknowledgments}

This work has been supported by the Czech Technical University in Prague under the grants No. SGS15/161/OHK3/2T/13 and SGS13/206/OHK3/3T/13.

\section{References}

[1] ETSI Standard. Digital Radio Mondiale (DRM); System Specification. ETSI ES 201980 V4.1.1, January 2014.

[2] iBiquity Digital Corporation. HD Radio Air Interface Design Description - Layer 1 AM. Doc. No. SY_IDD_1012s rev. F, August 2011.

[3] LIU, W., LAU, J., CHENG, R. Considerations on applying OFDM in a highly efficient power amplifier. IEEE Transactions on Circuits Systems II, 1999, vol. 46, no. 11 , p. 1329-1336. DOI: 10.1109/82.803472

[4] HOVERSTEN, J., SCHAFER, S., ROBERG, M., et al. Codesign of PA, supply, and signal processing for linear supplymodulated RF transmitters. IEEE Transactions on Microwave Theory and Techniques, 2012, vol. 60, no. 6, p. 2010-2020. DOI: 10.1109/TMTT.2012.2187920

[5] SEbASTiAn, J., MiAJA, P. F., GONZALEZ, F. J. O., et al. Design of a two-phase buck converter with fourth-order output filter for envelope amplifiers of limited bandwidth. IEEE Transactions on Power Electronics, 2014, vol. 29, no. 11, p. 5933-5948. DOI: 10.1109/TPEL.2013.2295035

[6] JOOSEUNG, K., DONGSU, K., YUNSUNG, C., et al. Analysis of envelope-tracking power amplifier using mathematical modeling. IEEE Transactions on Microwave Theory and Techniques, 2014, vol. 62, no. 6, p. 1352-1362. DOI: 10.1109/TMTT.2014.2321356

[7] HARRIS CORP. Advances in AM Modulation Techniques to Improve Digital Transmission of HD Radio and DRM. Harris Broadcast Communications, February 2004

[8] TRANSRADIO AG. $750 \mathrm{~kW}$ Solid State LF Broadcast Transmitter TRAM 750 LS LCD. TRANSRADIO Sender Systeme Berlin AG, March 2013.

[9] KAHN, L. R. Single sideband transmission by envelope elimination and restoration. In Proceedings of the IRE, 1952, vol. 40, no. 7, p. 803-806. DOI: 10.1109/JRPROC.1952.273844
[10] VASIĆ, M., GARCIA, O., OLIVER, J. A., et al. High efficiency power amplifier based on envelope elimination and restoration technique. In IEEE Energy Conversion Congress and Exposition (ECCE), 2010, p. 3833-3840. DOI: 10.1109/ECCE.2010.5617763

[11] WANG, F., KIMBALL, D. F., POPP, J. D., et al. An improved poweradded efficiency $19-\mathrm{dBm}$ hybrid envelope elimination and restoration power amplifier for $802.11 \mathrm{~g}$ WLAN applications. IEEE Transactions on Microwave Theory and Techniques, 2006, vol. 54, no. 12, p. 4086-4099. DOI: 10.1109/TMTT.2006.885575

[12] NESIMOGLU, T., MORRIS, K. A., PARKER, S. C., et al. Improved EER transmitters for WLAN. In IEEE Radio and Wireless Symposium, 2006, p. 239-242. DOI: 10.1109/RWS.2006.1615139

[13] BADAROU, A., REED, S., NDONG, D., et al. Power amplifier design challenges in UHF and VHF transmitters. In IEEE 11th International New Circuits and Systems Conference (NEWCAS), Paris, June 2013, p. 1-4. DOI: 10.1109/NEWCAS.2013.6573615

[14] ROHDE \& SCHWARZ GMBH \& CO. KG R\&S THU9/R\&S THV Liquid-cooled Transmitter Families, Efficiency Redefined. Rohde \& Schwarz GmbH \& Co. KG, Munich, Germany, February 2015.

[15] GODBOLE, B. B., NIKAM, R. H. FPGA implementation of CORDIC algorithm used in DDS based modulators. International Journal of Advanced Research in Computer and Communication Engineering, Jan. 2015, vol. 4, no. 1, p. 94-97. DOI: 10.17148/IJARCCE.2015.4119

[16] DOBEŠ, J. Using Volterra series for an estimation of fundamental intermodulation products. Radioengineering, 2008, vol. 17, no. 4, p. 59-64. WOS: 000262104800008

[17] RABB, F. H. Intermodulation distortion in Kahn-technique transmitters. IEEE Transactions on Microwave Theory and Techniques, Dec. 1996, vol. 44, no. 12, p. 2273-2278. DOI: 10.1109/22.556466

[18] DOBEŠ, J. Advanced types of the sensitivity analysis in frequency and time domains. AEU - International Journal of Electronics and Communications, 2009, vol. 63, no. 1, p. 52-64. DOI: 10.1016/J.AEUE.2007.10.008

[19] RUDOLPH, D. Out-of-band emissions of digital transmissions using Kahn EER technique. IEEE Transactions on Microwave Theory and Techniques, 2002, vol. 50, no. 8, p. 1979-1983. DOI: $10.1109 /$ TMTT.2002.801349

[20] RUDOLPH, D. Kahn EER technique with single-carrier digital modulations. IEEE Transactions on microwave theory and techniques, 2003, vol. 51, no. 2, p. 548-552. DOI: 10.1109/TMTT.2002.807810

[21] MIAJA, P. F., SEBASTIAN, J., MARANTE, R., et al. A Linear assisted switching envelope amplifier for a UHF polar transmitter. IEEE Transactions on Power Electronics, 2014, vol. 29, no. 4, p. 1850-1861. DOI: 10.1109/TPEL.2013.2270916

[22] MATĚJKA, Š. All digital FPGA based PWM modulator for radio frequency transmitters. In Konference Radioelektronika 2015, Pardubice (Czech Republic), Apr. 2015, p. 244-247. DOI: 10.1109/RADIOELEK.2015.7129022

[23] FEDORENKO, P. Phase Distortion in Envelope Elimination and Restoration Radio Frequency Power Amplifiers. Ph.D. Thesis, Georgia Institute of Technology, August 2009.

[24] MIDDLETON, D. An Introduction to Statistical Communication Theory. New York: McGraw-Hill, 1960.

[25] LATHI, B. P. Modern Digital and Analog Communication Systems. 3rd ed. New York, Oxford: Oxford University Press, 1998. ISBN: 0195110099

[26] PEEBELS, P. Z. Probability, random Variables and Random Signal Principles. 4th ed. McGraw-Hill Publishing Company, 2001. ISBN: 0073660078 
[27] BANELLI, P., CACOPARDI, S. Theoretical analysis and performance of OFDM signals in nonlinear AWGN channels. IEEE Transactions on Communications, 2000, vol. 48, no. 3, p. 430-441. DOI: $10.1109 / 26.837046$

[28] EBU TECHNICAL REPORT. Technical Bases for DRM Services Coverage Planning. EBU - Tech 3330, Geneva, June 2008.

[29] ETSI Standard. Electromagnetic compatibility and Radio spectrum Matters (ERM); Transmitting Equipment for the Digital Radio Mondiale (DRM) Broadcasting Service; Part 1: Technical Characteristics and Test Methods. ETSI EN 302 245-1 V1.1.1, January 2005.

[30] ETSI Standard. Electromagnetic Compatibility and Radio Spectrum Matters (ERM); Transmitting Equipment for the Digital Radio Mondiale (DRM) Broadcasting Service; Part 2: Harmonized EN under Article 3.2 of the R\&TTE Directive. ETSI EN 302 245-2 V1.1.1, January 2005.

[31] COLANTONIO, P., GIANNINI, F., LIMITI, E. High Efficiency RF and Microwave Solid State Power Amplifiers. John Wiley \& Sons, 2009. ISBN: 978-0-470-51300-2

\section{About the Authors ...}

Štěpán MATĚJKA was born in 1973. He received his M.Sc. and Ph.D. from the Faculty of Electrical Engineering, Czech Technical University in Prague in 1996 and 2004, respectively. From 1996 to 1997 he was with Elektrotechnika Tesla Kolín, Czech Republic, where he worked as a development engineer and cooperated on finalization and deployment of GPS based on board navigation system for the Czech Army fighter planes. In 2000, he affiliated the Czech Technical University in Prague as a radio frequency measurement and design tutor. His research interests include radio frequency circuits and systems design and measurement, digital signal processing, and radio transmitter and receiver systems. 